\title{
Artificial neural-network technique for precipitation nowcasting from satellite imagery
}

\author{
G. Rivolta $^{1}$, F. S. Marzano ${ }^{1,2}$, E. Coppola ${ }^{1}$, and M. Verdecchia ${ }^{1}$ \\ ${ }^{1}$ Centro di Eccellenza CETEMPS, Università dell'Aquila, L'Aquila, Italy \\ ${ }^{2}$ Dipartimento di Ingegneria Elettronica, Università di Roma "La Sapienza", Rome, Italy
}

Received: 11 November 2005 - Revised: 16 December 2005 - Accepted: 29 December 2005 - Published: 2 February 2006

\begin{abstract}
The term nowcasting reflects the need of timely and accurate predictions of risky situations related to the development of severe meteorological events. In this work the objective is the very short term prediction of the rainfall field from geostationary satellite imagery entirely based on neural network approach. The very short-time prediction (or nowcasting) process consists of two steps: first, the infrared radiance field measured from geostationary satellite (Meteosat 7 ) is projected ahead in time ( $30 \mathrm{~min}$ or $1 \mathrm{~h}$ ); secondly, the projected radiances are used to estimate the rainfall field by means of a calibrated microwave-based combined algorithm. The methodology is discussed and its accuracy is quantified by means of error indicators. An application to a satellite observation of a rainfall event over Central Italy is finally shown and evaluated.
\end{abstract}

\section{Introduction}

Nowcasting of rainfall from satellite imagery is becoming an important issue for several applications, mainly related to civil protection alarming, but also to mesoscale system atmospheric dynamics (Marzano et al., 2002; Grimes et al., 2003; Coppola et al., 2005). Multiple space and time scales can be taken into account, as well as different data sources and objectives. The term "nowcasting" should be intended, in this context, as the ability to predict the evolution of the geophysical field of interest from satellite imagery at very short-term time scales. The satellite measurements implicitly define the time and space sampling. For a rapidly varying field such as rainfall, high repetition times, such as those available from geostationary satellites, are essential. On the other hand, the accuracy of the nowcasted field is strictly related to the correlation of the measured remotely sensed data with the rainfall field itself.

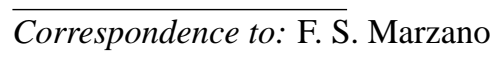

(marzano@die.uniroma1.it)
Several rain retrieval techniques have been proposed on the basis of image processing approaches and infrared (IR) radiometry data acquired from geostationary platforms (Hsu et al., 1997; Miller et al., 2001; Grimes et al., 2003). These approaches denote some inherent limitations due to the use of IR radiances, which are measurements poorly correlated with rainfall-forming processes. In this respect, microwave (MW) radiometric data available from sun-synchronous platforms can give best results being physically correlated with rain below the cloud (Kummerow et al., 1995; Levizzani et al., 1996; Tapiador et al., 2004; Marzano et al., 2005). From a meteorological point of view, visible (VIS) and IR radiometers can give information on cloud top layers. On the other hand, MW radiometers can detect cloud structure and rainrate since MW brightness temperatures (TB's) are fairly sensitive to liquid and ice hydrometeors (Ferraro, 1997). Regarding the platforms, Geosynchronous Earth Orbit (GEO) satellites can ensure a coverage with a high temporal sampling, while Low Earth Orbit (LEO) satellites have the advantage to enable the use of MW sensors, but with the major drawback of low temporal sampling. Therefore, LEOMW and GEO-IR radiometry are clearly complementary for monitoring the Earth's atmosphere and a highly variable phenomenon such as precipitation. The IR radiances from geostationary images have been properly calibrated using MWbased combined algorithms (Turk et al., 2000; Bellerby et al., 2000; Marzano et al., 2004). MW data can be extracted from the Special Sensor Microwave Imager (SSM/I) sensors, but any rain estimation source may be foreseen.

Rainfall nowcasting by remote sensing imagery has been approached by means of numerous techniques in the last decade (e.g., Dell'Acqua and Gamba, 2003). The following nowcasting methods may be classified among standard, or in this paper "conventional", techniques: i) persistence method, which assumes that each pixel in the forecasted satellite image is associated to the same value as in the previous image; ii) steady-state method, which assumes that the structures in the image are transported but unchanged in size and intensity (the forecasted image is the latest available satellite image 


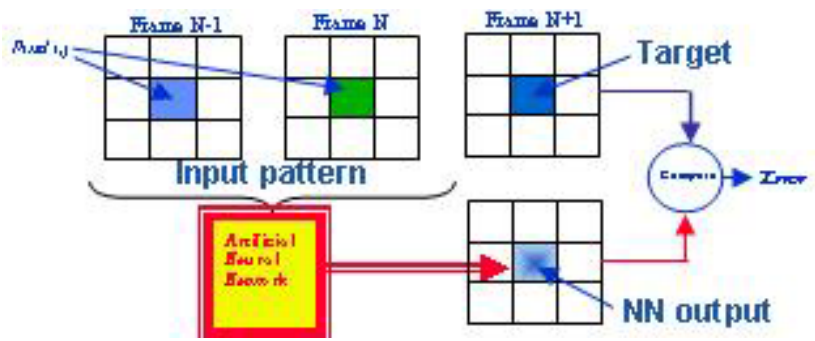

Fig. 1. Neural network (NN) inputs and outputs during the training phase.

translated by a suitable motion vector); iii) linear method, consisting in linearly extrapolating the future value of a pixel in the satellite frame from the latest two values available. Each of these conventional nowcasting methods shows a performance that depends on the weather conditions in the considered region. None of the three can be assumed as the best method for all possible conditions. Neural networks (NN) present several advantages with respect to conventional statistical techniques (Tapiador et al., 2004a, b; Marzano et al., 2005). Their main feature is the ability to map input data to output data to any degree of non-linearity. It is well known that NNs exhibit the capacity to learn and to represent highly non-linear functional.

In this work a special attention has been devoted to select the input space-time features in order to optimize the NN methodology for rainfall nowcasting from satellite imagery. For a quantitative evaluation, various performance indexes have been applied. Application to selected case studies will be discussed in order to show the potential of the proposed satellite-based nowcasting technique. The comparison of these preliminary results of the different techniques clearly shows the better nowcasting capability of the neural techniques with respect to the conventional techniques.

\section{Satellite nowcasting of IR radiance field}

As a source of geostationary satellite imagery, Meteosat-7 has been here considered over areas of Southern Europe. The IR Meteosat- 7 images considered here are composed by $547 \times 298$ pixels, corresponding roughly to longitudes ranging from $0^{\circ}$ to $22^{\circ} \mathrm{E}$ and latitudes ranging from $36^{\circ}$ to $48^{\circ} \mathrm{N}$. Each pixel can be approximated as a square of $5 \times 5 \mathrm{~km}^{2}$. Three conventional forecast methods have been considered for comparison and are briefly described below.

\subsection{Conventional nowcasting methods}

i) The first technique is the persistence method. This method assumes that each pixel in the forecasted satellite image is associated to the same value of the previous image. This means that if the latest available (or current) image is frame $\mathrm{N}$, the next satellite image, or frame $\mathrm{N}+1$, is assumed to be equal to frame $\mathrm{N}$. ii) The second technique is the steady-state method. This method assumes that the structures in the satellite frame $\mathrm{N}$ are transported but unchanged in size and intensity. Frame $\mathrm{N}+1$ (or forecasted image) is assumed to be equal to the last available satellite image, translated by a suitable motion vector. The proper displacement vector is found by calculating the cross-correlation index between frames $\mathrm{N}$ and $\mathrm{N}-1$ for all the possible shifts of one of the two images with respect to the other, within a range of $+/-8$ pixels. The values found for each component of the vector are in the range $0-3$ pixel.

iii) The third technique is the linear method. This method consists in linearly extrapolating the future value of a pixel. The IR temperature associated to each pixel in the frame $\mathrm{N}+1$ is linearly extrapolated from the values associated to the same pixel in the last two available images (frames $\mathrm{N}$ and N-1).

These conventional methods exhibit a performance that depends on the weather conditions in the considered region. None of the three can be assumed as the best method for all possible conditions.

\subsection{Neural-network nowcasting method}

It is well known that NNs exhibit the capacity to learn and to represent highly non-linear functional. A neural network, properly configured, can be regarded to as a universal function approximator (Hecht-Nielsen, 1991). In general, NNs provide a powerful methodology to predict temporal series of random variables.

In this work NNs are trained to forecast the IR temperature value associated to a certain pixel of the satellite image, starting from the measured values in a region around that pixel in the previous satellite images. To this aim, a great number (some tens of thousands) of input pattern/target pairs are provided to the network during the training phase. Figure 1 shows an example in which the input pattern is a vector of 18 elements, corresponding to the square areas of 9 pixels centered on the pixel $i, j$, both in frame $\mathrm{N}$ and in frame $\mathrm{N}-1$. The associated target provided to the network during the training phase is the IR temperature of the pixel $i, j$ in the frame $\mathrm{N}+1$.

In this example the input pattern is built considering the neighbouring region around the pixel to be forecasted in the last two available satellite images. In general, the extension of the region around the pixel to be forecasted and the number of previous satellite images can be varied in order to optimize the network response.

For tracking cloud structures, at the scale considered here, a typical wind velocity of $20 \mathrm{~km} \mathrm{~h}^{-1}$ can be assumed. This means that in $30 \mathrm{~min}$ a certain cloud structure is expected to be found within a radius of $10 \mathrm{~km}$. The greater is the number of past frames taken into account, the greater should be the extension of the region around the considered $i, j$ pixel for building the input pattern. This means that the local spatial scale has to be established according to the considered time lag. 


\section{The neural network architecture}

The neural nowcasting technique proposed here is based on the feedforward multi-layer structure. This type of network is characterized by the forward propagation of the input information through the various layers. All neurons in a layer are connected to all neurons in the adjacent layers through unidirectional links. These links are represented by the synaptic weights. The synaptic weights act as signal multipliers on the corresponding links (interconnections). For example, in the four layers network showed in Fig. 2, the neurons are grouped in sequentially connected layers; the layers are numbered $0,1,2$ and 3 . The neurons of layer 0 (or input layer) do not perform computation, but only feed input signals to the neurons of layer 1 (or first hidden layer). The last layer (layer 3 ) is the output layer. In this work the output layer has only one neuron since the objective is (as stated before) to forecast the IR temperature value associated to a certain pixel $i, j$.

The layers between the input and the output layer are called hidden layers. It has been shown theoretically that a three layers feed-forward neural network is sufficient to map any continuous function (Hecht-Nielsen, 1991). Each neuron is connected to all neurons of the two adjacent layers and not to other neurons. Connections between neurons of the same layer are not permitted and the input signal proceeds from the input to the output layer and never in the opposite direction. Each neuron is characterized by one output and many inputs, which are the outputs of the neurons in the preceding layer. The output of the neuron $j$ in the layer $s$ is:

$o_{j}^{s}=\psi_{j}^{s}\left[\sum_{i=1}^{N_{s-1}} W_{j i}^{s} o_{i}^{s-1}\right]$

where $\Psi_{j}^{s}$ is the non-linear bounded activation function of the neuron and $W_{j i}^{s}$ represent the synaptic weight connecting the input of the $j$-th neuron in the layer $s$ to the output of the $i$-th neuron in the layer $s-1$. In this work, a log-sigmoid activation function is used for all neurons.

The $N N$ is trained minimizing the error function $E$ by the steepest-descent gradient back-propagation algorithm. Several tens of thousands of input/output patterns are provided to the network during the training phase. The synaptic weights are updated during the training according to the following equation

$\Delta W_{i j}(t)=-\alpha \frac{\partial E}{\partial W_{i j}}+\beta \Delta W_{i j}(t-1)$

where $\alpha$ and $\beta$ are the learning rate and the momentum parameters, respectively, $E$ the error function, and $t$ is the training step or epoch.

\section{Results}

Several configurations of feed-forward networks have been trained on selected IR satellite images from the 48 frames sequence of 24 January 2003. In order to introduce in the

\section{Multilayer Artificial Neural Network}

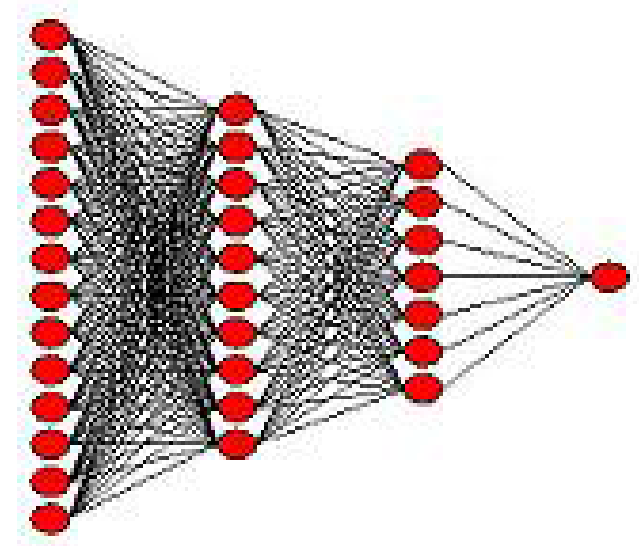

Fig. 2. Example of feed-forward neural network.

network the information of the different phases of the meteorological process, the pairs of input and output examples utilized for the training phase have been chosen at a temporal distance of $6 \mathrm{~h}$, for a total of 4 series of Meteosat images. The frames 4, 16, 28 and 40 have been used as target frames.

Different number of training patterns, synaptic weights, neighbours and preceding frames have been used for training several network configurations.

The resulting trained networks have been tested on two different sequences of frames extracted from 24 and 25 January, for the prediction of the IR satellite image $\mathrm{N}+1$, being $\mathrm{N}$ the current or latest available Meteosat image. The performances of the different configurations are evaluated by statistical indexes and compared to the performances of conventional methods. The error bias, root mean square error (rmse) and correlation index (corr) are defined as follows:

$$
\begin{aligned}
& \text { bias }=\frac{1}{N} \sum_{i=1}^{N} x_{i}-y_{i} \\
& \text { rmse }=\left(\frac{1}{N} \sum_{i=1}^{N}\left(x_{i}-y_{i}\right)^{2}\right)^{1 / 2} \\
& \text { corr }=\frac{\sum_{i=1}^{N}\left(x_{i}-\bar{x}\right)\left(y_{i}-\bar{y}\right)}{\left(\sum_{i=1}^{N}\left(x_{i}-\bar{x}\right)^{2} \sum_{i=1}^{N}\left(y_{i}-\bar{y}\right)^{2}\right)^{1 / 2}}
\end{aligned}
$$

where $x$ and $y$ are the forecasted and measured values, respectively.

The best performing NN configuration in the prediction of frame $\mathrm{N}+1$ (half an hour ahead) has been trained and tested for the prediction of the satellite image $\mathrm{N}+2$ ( $1 \mathrm{~h}$ ahead). In Fig. 3 the correlation index results computed between the 


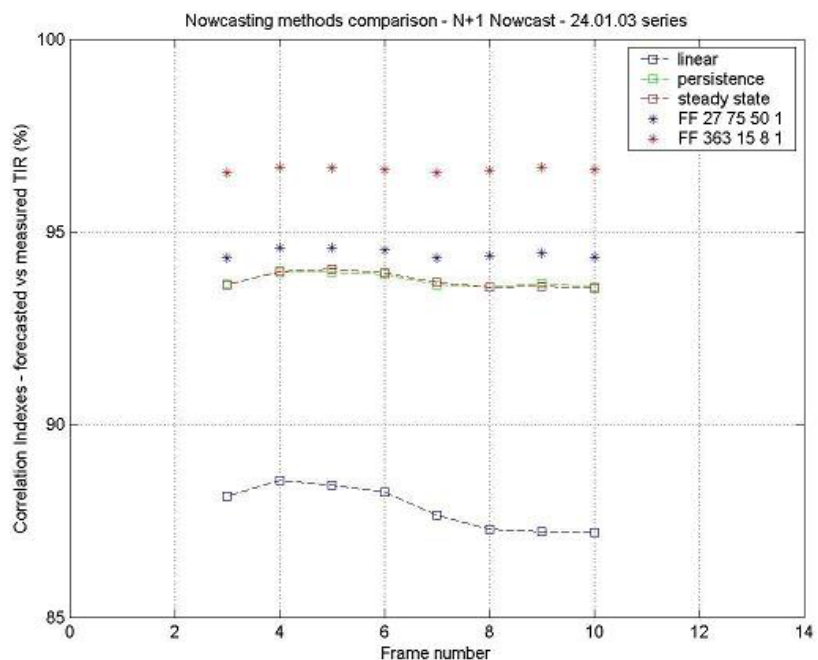

Fig. 3. Correlation indexes $(N+1)$ comparison on 24 Jan 2003 sequence.

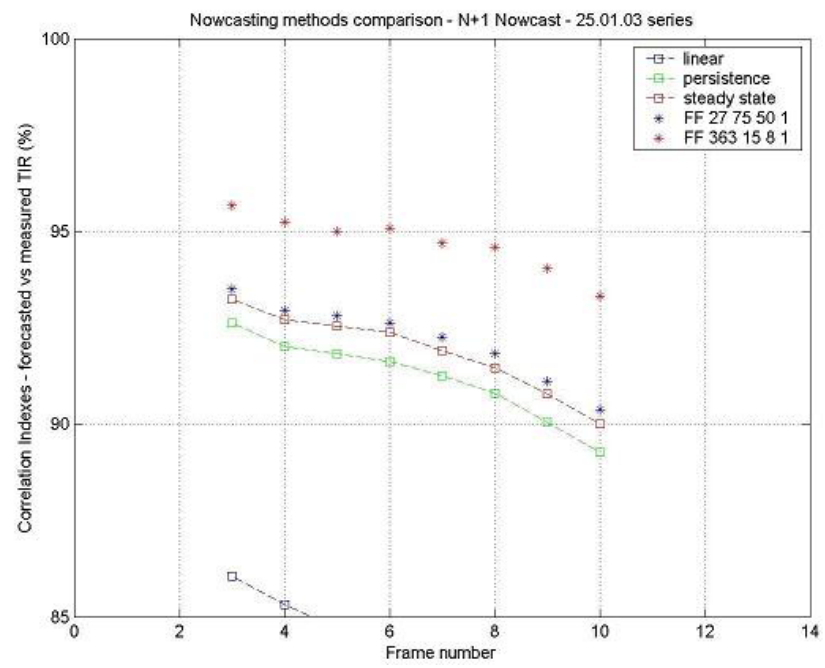

Fig. 4. Correlation indexes (N+1) comparison on 25 Jan 2003 sequence.

forecasted and true image in the case of two different neural configurations and conventional methods are reported.

The results are similar for the steady-state and the persistence method, while the linear method performs quite poorly with respect to the other two methods. The correlation indexes of images predicted with the neural configurations, are greater than those obtained through the conventional methods. The FF_27_75_50_1 is a feed-forward network with 27 elements in the input pattern, 75 neurons in the first hidden layer, 50 neurons in the second hidden layer and 1 neuron in the output layer. This configuration utilizes 1 level of neighbours (that is 9 pixels) from the last three previous frames. The FF_363_15_8_1 utilizes five levels of neighbours (121 pixels) from three previous frames. For different network configurations the number of neurons in the hidden layers is established so that the ratio between the training exam-

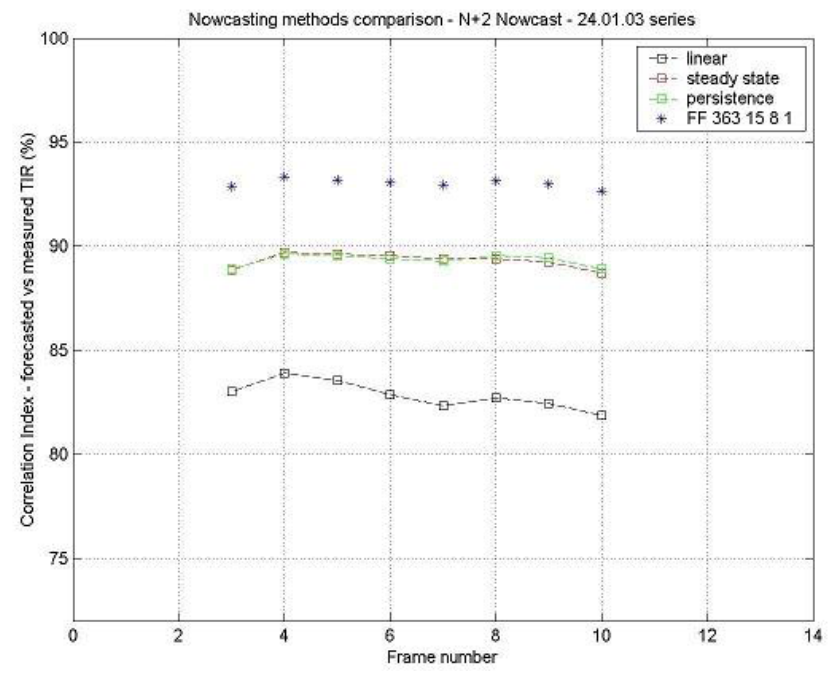

Fig. 5. Correlation indexes $(\mathrm{N}+2)$ comparison on 24 Jan 2003 sequence.

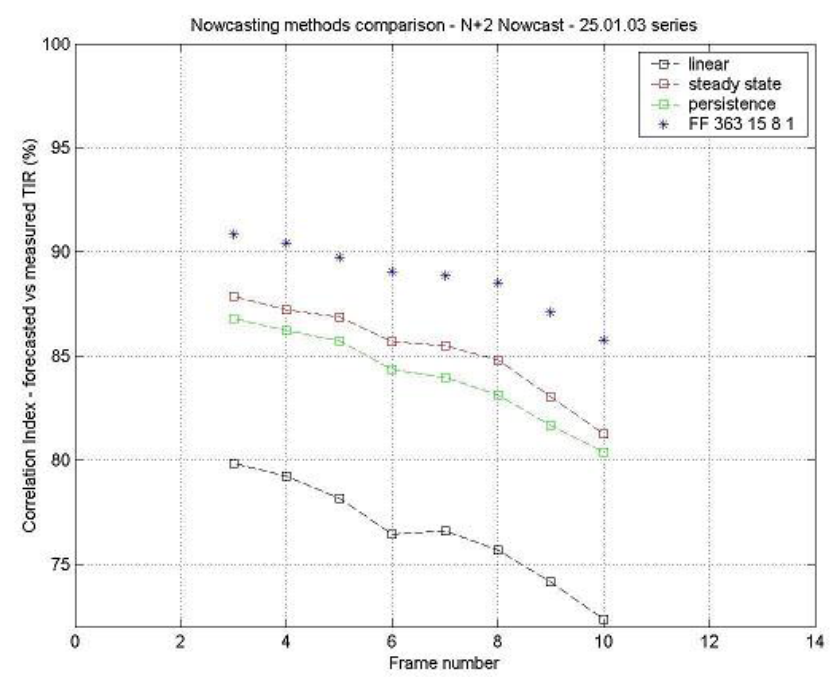

Fig. 6. Correlation indexes $(\mathrm{N}+2)$ comparison on 25 Jan 2003 sequence.

ples and the number of weights to be optimized remains unchanged for all the configurations.

The different results obtained by two FF networks are due to the different considered area around the pixel.

The configuration FF_363_15_8_1 has also been trained and tested for the prediction of frame N+2 (1 h ahead). Figures 5 and 6 show the correlation indexes obtained by neural predictions compared to conventional methods, for 24 and 25 January sequences, respectively.

The positive margin of neural nowcasting with respect to conventional nowcasting in terms of correlation index results increases in the prediction of frame $\mathrm{N}+2$, ranging between 3.7 and $4.7 \%$.

The results in terms of rmse and bias also confirm that the best performing neural configuration is the FF_363_15_8_1. 


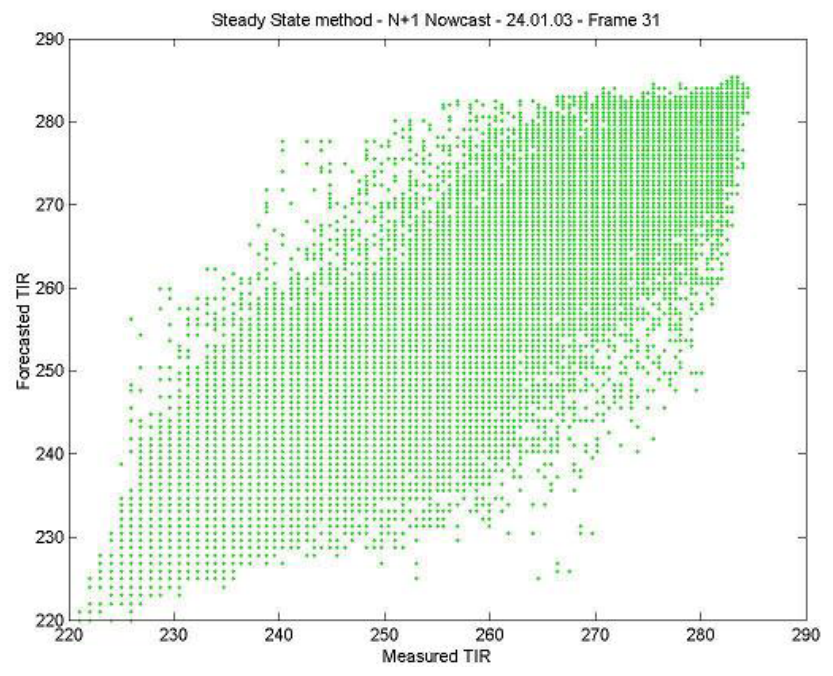

Fig. 7. Steady-state nowcasting (N+1) of the frame 31, 24 Jan 2003.

In Tables 1 to 4 the performance indexes related to the sequences of 24 and 25 January are reported for the neural nowcasting and the steady-state method.

In Figs. 7 and 8 an example of scatterplot related to the best conventional method (steady-state) is compared to the corresponding scatterplot related to the best neural configuration, respectively. The results shown so far indicate that a properly configured and trained NN can significantly outperform the conventional nowcasting methods.

Nevertheless, the performance of a NN configuration depends on the choice of the number of previous frames and the number of neighbours. A further improvement in the performance of a certain neural configuration is expected if a wider set of input patterns, representative of different meteorological situations, is provided to the network in the training phase.

An example of neural prediction of an IR temperature field by means of the FF_363_15_8_1 configuration is shown in Fig. 9. The left panel of Fig. 9 shows the observed Meteosat image, whereas the right panel the nowcasted image derived from the NN approach. The comparison shows an excellent agreement between the two radiance fields, even in the region of low equivalent temperatures, which correspond to cloud and rain coverage.

\section{Rainfall nowcasted field estimation algorithm using MW and IR sensors}

The predicted IR field can be used as input to any rainfall estimation algorithm capable to process IR satellite data. For producing rainfall field estimation from the nowcasted IR temperature field, the NEREMIS algorithm has been considered (Grimes et al., 2003; Marzano et al., 2005; Coppola et al., 2005). An example of estimated rainfall field is shown in Fig. 10. The image on the left represents the rain field estimated by the NEREMIS technique from the real IR tem-

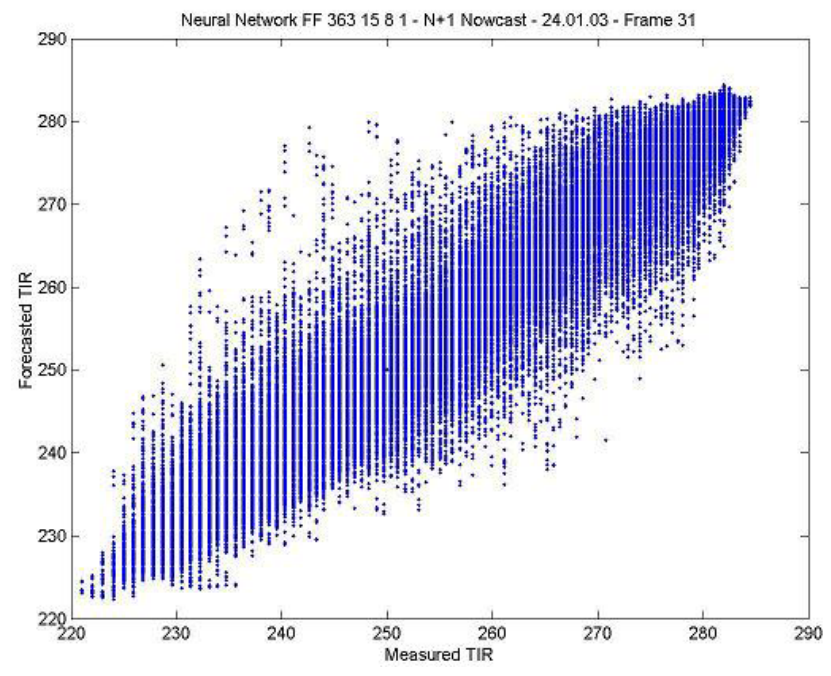

Fig. 8. Neural nowcasting (N+1) of the frame 31, 24 Jan 2003.

perature field as observed from Meteosat 7. On the right the rainfall field estimated from the neural prediction of the IR temperature field is shown. The rmse is about $0.4 \mathrm{~mm} \mathrm{~h}^{-1}$ when selecting the rain areas.

\section{Summary and future developments}

The comparison of different techniques for the nowcasting of satellite IR brightness temperature images shows that the neural approach is definitely performing better than any conventional method considered in the comparison. Both the predictions at $30 \mathrm{~min}$ and $1 \mathrm{~h}$ have been compared, showing an increased positive margin in terms of correlation indexes for the neural prediction of the frame $\mathrm{N}+2$.

Several four layer feed-forward neural configurations have been tested, varying the number of preceding frames and the number of levels of neighbour pixels. A further increase in the nowcasting performance of a neural configuration is expected if a wider set of input patterns, representative of different meteorological situations, is provided to the network in the training phase. For rainfall field nowcasting, the predicted IR temperature field has been used as input for a combined IR/MW estimation algorithm. Open issues regard the error budget evaluation for the MW-IR combined rain estimation algorithms (over land) and the network optimal design for an operational use.

Acknowledgements. This work has been partially funded by Regione Abruzzo, Italy and the RiskAWARE project within the INTERREG-IIIB CADSES framework.

Edited by: V. Kotroni and K. Lagouvardos

Reviewed by: anonymous referee 
Table 1. Nowcasting performance indexes $(\mathrm{N}+1)$ on Jan 24, 2003 sequence for the neural configuration FF_363_15_8_1.

\begin{tabular}{ccccccccc}
\hline \multicolumn{8}{c}{ FF_363_15_8_1-24.01.03 } \\
\hline Frame N & 3 & 4 & 5 & 6 & 7 & 8 & 9 & 10 \\
bias & -0.199 & -0.303 & -0.289 & -0.408 & -0.384 & -0.376 & -0.351 & -0.374 \\
rmse & 4.216 & 4.185 & 4.209 & 4.243 & 4.269 & 4.239 & 4.168 & 4.172 \\
corr & 96.54 & 96.67 & 96.66 & 96.61 & 96.54 & 96.59 & 96.68 & 96.61 \\
\hline
\end{tabular}

Table 2. Nowcasting performance indexes $(\mathrm{N}+1)$ on Jan 25, 2003 sequence for the neural configuration FF_363_15_8_1.

\begin{tabular}{ccccccccc}
\hline \multicolumn{8}{c}{ FF_363_15_8_1-25.01.03 } \\
\hline Frame N & 3 & 4 & 5 & 6 & 7 & 8 & 9 & 10 \\
bias & -0.219 & -0.263 & -0.175 & -0.231 & -0.255 & -0.235 & -0.173 & -0.197 \\
rmse & 4.516 & 4.709 & 4.814 & 4.736 & 4.864 & 4.867 & 5.048 & 5.315 \\
corr & 95.68 & 95.25 & 95.00 & 95.08 & 94.68 & 94.58 & 94.04 & 93.32 \\
\hline
\end{tabular}

Table 3. Nowcasting performance indexes $(\mathrm{N}+1)$ on 24 Jan 2003 sequence for the steady-state method.

\begin{tabular}{ccccccccc}
\hline \multicolumn{8}{c}{ Steady-state $-24.01 .03$} \\
\hline Frame N & 3 & 4 & 5 & 6 & 7 & 8 & 9 & 10 \\
bias & 0.011 & -0.036 & -0.014 & -0.130 & -0.185 & -0.148 & -0.125 & -0.163 \\
rmse & 5.778 & 5.665 & 5.677 & 5.735 & 5.826 & 5.863 & 5.836 & 5.827 \\
corr & 93.62 & 93.97 & 94.04 & 93.93 & 93.68 & 93.56 & 93.59 & 93.54 \\
\hline
\end{tabular}

Table 4. Nowcasting performance indexes (N+1) on 25 Jan 2003 sequence for the steady-state method.

\begin{tabular}{ccccccccc}
\hline \multicolumn{8}{c}{ Steady-state $-25.01 .03$} \\
\hline Frame N & 3 & 4 & 5 & 6 & 7 & 8 & 9 & 10 \\
bias & 0.058 & 0.058 & 0.104 & 0.044 & 0.015 & 0.020 & 0.076 & 0.082 \\
rmse & 5.747 & 5.943 & 5.999 & 6.031 & 6.141 & 6.250 & 6.445 & 6.665 \\
corr & 93.23 & 92.70 & 92.55 & 92.38 & 91.90 & 91.46 & 90.78 & 90.01 \\
\hline
\end{tabular}

\section{References}

Bellerby, T., Todd, M., Kniveton, D., and Kidd, C.: Rainfall Estimation from a Combination of TRMM Precipitation Radar and GOES Multispectral Satellite Imagery through the Use of an Artificial Neural Network, J. Appl. Meteor., 39, 2115-2128, 2000.

Coppola, E., Grimes, D. I. F., Verdecchia, M., Visconti, G.: Validation of improved TAMANN neural network for operational satellite-derived rainfall estimation in Africa, J. Appl. Meteor., accepted, 2005.

Dell'Acqua, F. and Gamba, P.: Pyramidal Rain Field Decomposition Using Radial Basis Function Neural Networks for Tracking and Forecasting Purposes, IEEE Trans. Geosci. Remote Sens., 41, 4, pp. 853, 2003.

Ferraro R. R.: SSM/I derived global rainfall estimates for climatological applications, J. Geophys. Res., 102, 16 715-16735, 1997.

Grimes, D. I. F., Coppola, E., Verdecchia, M., and Visconti, G.:
A neural network approach to real time rainfall estimation for Africa using satellite data, J. Hydrometeor., 6, 4, 1119-1133, 2003.

Hecht-Nielsen, R.: Neurocomputing, Addison Wesley Publishing Company, 1991.

Hsu, K. L., Gao, X., Sorooshian, S., and Gupta, H. V.: Precipitation estimation from remotely sensed information using artificial neural networks, J. Appl. Meteor., 36, 1176-1190, 1997.

Kummerow, C. and Giglio, L.: A method for combining passive microwave and infrared rainfall observations, J. Atmos. Oceanic Tecnol., 12, 33-45, 1995.

Levizzani, V., Porcù, F., Marzano, F. S., Mugnai, A., Smith, E. A., and Prodi, F.: Investigating a SSM/I microwave algorithm to calibrate METEOSAT infrared instantaneous rain-rate estimates, Meteorol. Appl., 3, 5-17, 1996.

Marzano, F. S., Turk, J., Ciotti, P., Di Michele, S., and Pierdicca, N.: Potential of combined spaceborne microwave and infrared ra- 
Mest th olemer bell

Observed TIR

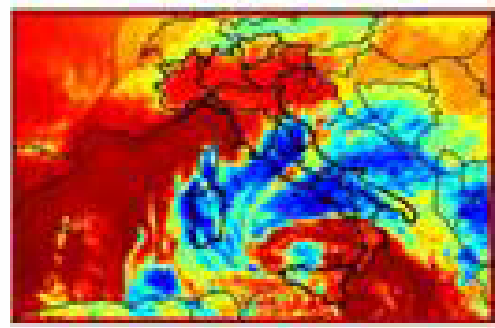

trame at $24-61-03$
Mxat Tb fineld Neural Nowesaling

\section{Predicted TIR}
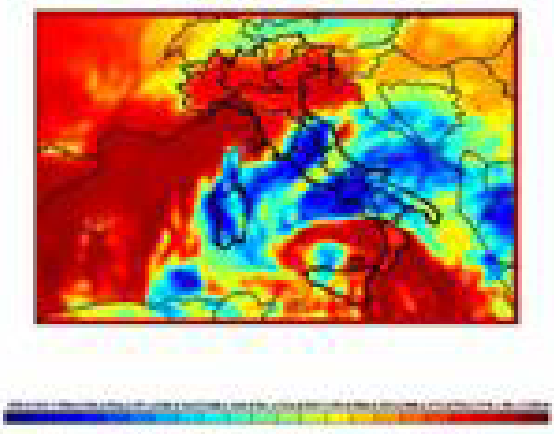

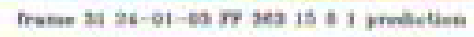

Fig. 9. An example of neural prediction vs observed TIR field.

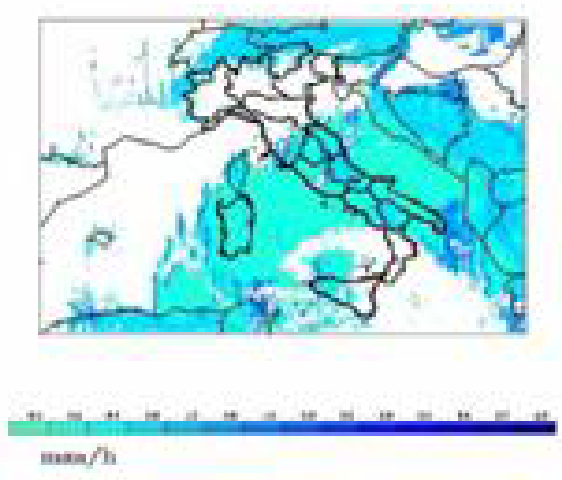

Fig. 10. Nowcasted rainfall field vs estimation from observed IR field.

diometry for near real-time rainfall attenuation monitoring along earth satellite, Int. J. Satell. Commun., 19, 4, 385-412, 2001.

Marzano, F. S., Mugnai, A., and Turk, J.: Precipitation retrieval from spaceborne microwave radiometers and combined sensors, in: Remote sensing of atmosphere and ocean from space, edited by: Marzano, F. S. and Visconti, G., Kluwer Academic Publ., Dordrecht (NL), pp. 107-126, 2002.

Marzano, F. S., Palmacci, M., Cimini, D., Giuliani, G., and Turk, J. F.: Multivariate Statistical Integration of Satellite Infrared and Microwave Radiometric Measurements for Rainfall Retrieval at the Geostationary Scale, IEEE Trans. Geosci. Remote Sens., 42, 4, 1018-1032, 2004.

Marzano, F. S., Cimini, D., Coppola, E., Verdecchia, M., Levizzani, V., Tapiador, F., and Turk, J. F.: Satellite radiometric remote sensing of rainfall fields: mult-isensor retrieval techniques at geostationary scale, Adv. Geosci., 2, 267-272, 2005.

Miller, S. W., Arkin, P. A., and Joyce, R.: A combined microwave infrared rain rate algorithm, Int. J. Remote Sens., 22, 3285-3307, 2001.

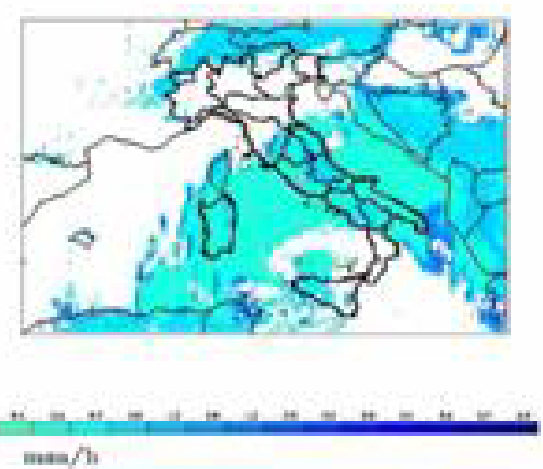

Tapiador, F. J., Kidd, C., Levizzani, V., and Marzano, F. S.: A neural networks-based PMW-IR fusion technique to derive half hourly rainfall estimates at $0.1^{\circ}$ resolution, J. Appl. Meteor., 43, 576594, 2004a.

Tapiador, F. J., Kidd, C., Hsu, K. L., and Marzano, F. S.: Neural Networks in Satellite Rainfall Estimation, Meteorol. Appl., 11, 1-9, 2004b.

Turk, J. F., Rohaly, G., Hawkins, J., Smith, E. A., Marzano, F. S., Mugnai, A., and Levizzani, V.: Meteorological applications of precipitation estimation from combined SSM/I, TRMM, and geostationary satellite data, in: Microwave Radiometry and Remote Sensing of the Environment, edited by: Pampaloni, P., VSP Int. Sci. Publisher, Utrecht (NL), pp. 353-363, 2000.

Vicente, G. A., Scofield, R. A., and Menzel, W. P.: The operational GOES infrared rainfall estimation technique, Bull. Amer. Meteor. Soc., 79, 1883-1898, 1998. 